\title{
Pancreatic Stellate Cell
}

National Cancer Institute

\section{Source}

National Cancer Institute. Pancreatic Stellate Cell. NCI Thesaurus. Code C107531.

A star-shaped, myofibroblast-like cell in the pancreas associated with tissue maintenance. When pancreatic stellate cells are activated in response to an injury, they proliferate and synthesize large amounts of extracellular matrix. Pancreatic cancer cells can increase the activation of pancreatic stellate cells, which leads to fibrosis. 